\title{
Modélisation phénoménologique du comportement de céramiques ferroélectriques et ferroélastiques
}

\author{
Mourad Elhadrouz ${ }^{a}$, Tarak Ben Zineb et Étienne Patoor \\ Laboratoire de Physique et Mécanique des Matériaux UMR CNRS 7554, École Nationale Supérieure d'Arts et Métiers, \\ 4 rue Augustin Fresnel, Metz Technopôle, 57078 Metz Cedex 3, France
}

Reçu le 25 février 2005, accepté le 21 mars 2005

\begin{abstract}
Résumé - Les céramiques piézoélectriques présentent un couplage électromécanique leur donnant la capacité de développer sous une contrainte mécanique une charge électrique proportionnelle à celle-ci et de se déformer en présence d'un champ électrique. Cette propriété est à la base des nombreuses applications telles que des actionneurs ou des capteurs. Pour des chargements plus importants, la réponse n'est plus linéaire. Ce comportement s'explique par la rotation de zones uniformément polarisées qui engendre une polarisation et une déformation irréversibles responsables des comportements ferroélectrique et ferroélastique. L'exploitation de ces comportements non-linéaires permet des déplacements plus importants mais elle est freinée par la complexité du comportement et l'absence d'outils de dimensionnement. Une modélisation phénoménologique décrivant la piézoélectricité, ferroélasticité et ferroélectricité est présentée. Des cyclages électriques ainsi que le phénomène de dépolarisation mécanique sont simulés sur la base de ce modèle.
\end{abstract}

Mots clés : Piézoélectricité / ferroélectricité / ferroélasticité

\begin{abstract}
Piezoceramics exhibit an electro-mechanical coupling which enables them to produce a proportional electric charge when subjected to a mechanical stress or to deform in presence of an electric field. This behaviour is used in many applications such as actuators or sensors. In the case of more severe loadings, the response is no longer linear and it is characterised by the switching of uniformly polarised domains. Thus, ferroelectricity and ferroelasticity induce irreversible polarisation and strain. This non-linear effect is desired for present days applications since greater values of displacements could be obtained. However, the complexity of the coupling has to be overcome to design structures involving such materials and some tools like finite element codes taking into account this specific behaviour are needed for this purpose. A constitutive model for ferroelectric and ferroelastic piezoceramics is presented. Uniaxial electro-mechanical loadings have been computed.
\end{abstract}

Key words: Piezoelectricity / ferroelectricity / ferroelasticity / electromechanical coupling / modeling

\section{Introduction}

Les céramiques piézoélectriques, tel le titano-zirconate de plomb (PZT), sont utilisées dans de nombreuses applications industrielles telles que les capteurs (effort, accélération ...), les actionneurs et de manière plus générale dans les applications dites « intelligentes » [1]. Le couplage électromécanique qui caractérise ces matériaux se traduit par leur capacité à développer sous une contrainte mécanique une charge électrique ou bien à se déformer en présence d'un champ électrique. Ce comportement linéaire est bien connu depuis de nombreuses

\footnotetext{
a Auteur correspondant :

mourad.elhadrouz@metz.ensam.fr
}

années. Cependant, il n'apparait que pour de faibles sollicitations. La demande industrielle actuelle tend vers l'exploitation du comportement non-linéaire apparaissant pour des chargements électro-mécaniques plus importants.

\section{Comportement d'une céramique ferroélectrique et ferroélastique}

\subsection{Structure cristallographique}

Le comportement des céramiques ferroélectriques et ferroélastiques repose sur leur structure cristallographique. Au-dessus de la température de Curie, 


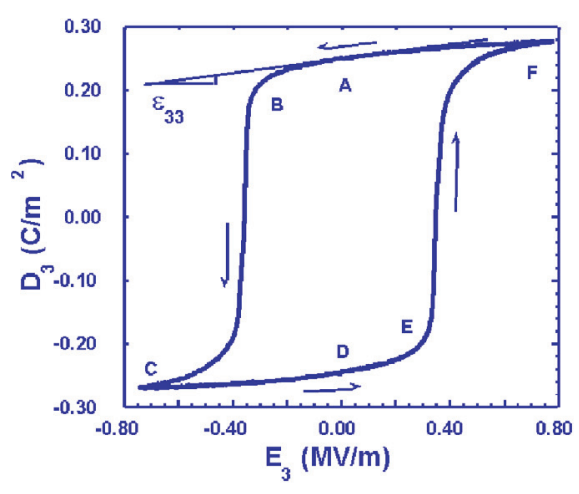

(a)

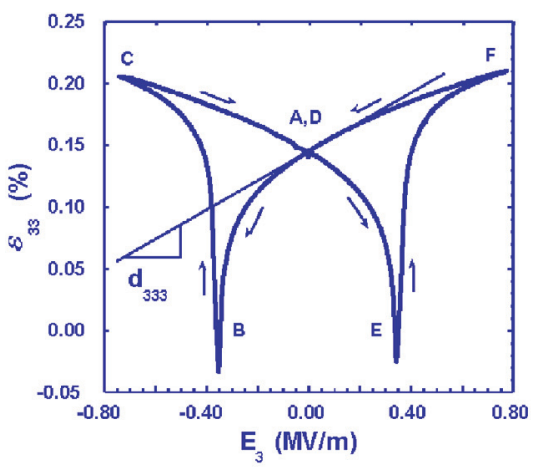

(b)

Fig. 1. Courbes d'hystérésis ferroélectriques : (a) évolution de la polarisation totale $\left(D_{3}\right)$ en fonction du champ électrique; (b) évolution de la déformation totale en fonction du champ électrique [2].

la structure cristallographique du titanate de plomb, un matériau ferroélectrique très utilisé, est cubique avec un atome de titane au centre de la maille élémentaire, un atome d'oxygène au centre de chaque face et un atome de plomb à chaque coin. Les symétries cubiques imposent la neutralité électrique. En dessous de cette température, la maille élémentaire devient quadratique et se polarise. La polarisation provient du déplacement relatif du barycentre des charges positives et de celui des charges négatives. Il en résulte un moment dipolaire. Ce moment dipolaire par unité de volume est la polarisation spontanée.

\subsubsection{Comportement ferroélectrique}

Un monocristal est composé de plusieurs mailles élémentaires. Lorsqu'il est refroidi sous la température de Curie, ces mailles élémentaires ne se polarisent pas tous spontanément dans la même direction. Les régions ayant la même polarisation sont qualifiées de domaines. Il en résulte que le monocristal ne présente pas de polarisation macroscopique. Par contre, l'application d'un champ électrique au-dessus d'une valeur appelée champ coercitif conduit au changement de direction de la polarisation individuelle de chaque maille élémentaire qui a tendance à s'aligner avec la direction du champ électrique. Le matériau présente alors une polarisation et une déformation rémanentes macroscopiques. C'est la réorientation ferroélectrique. Une fois le matériau polarisé, celui-ci est piézoélectrique (Fig. 1).

\subsubsection{Comportement ferroélastique}

L'application d'une contrainte de compression de direction opposée à celle de la polarisation induit une réorientation ferroélastique. Pour de faibles valeurs de contrainte, la réponse est élastique. Pour des valeurs plus importantes, la polarisation tourne. Cela introduit une déformation rémanente qui subsiste quand la contrainte s'annule. Après cette réorientation, il y a saturation et la réponse est à nouveau élastique. Il est possible d'observer une déformation rémanente mais également une baisse de la polarisation rémanente si le matériau est initialement polarisé. Ce phénomène est celui de la dépolarisation mécanique (Fig. 2).

L'ensemble de ces comportements est décrit par le modèle présenté par la suite [3].

\subsection{Modélisation}

\subsubsection{Thermodynamique [4]}

On postule qu'il existe un potentiel thermodynamique duquel les relations d'état découlent. Les états électrique et mécanique de la céramique peuvent être décrits par deux types de variables: les variables observables d'une part et les variables internes d'autre part. Les variables observables sont la température $T$, la déformation totale $\varepsilon$, le déplacement électrique $\vec{D}$. Une partition de la déformation totale $\varepsilon$ et du déplacement électrique $\vec{D}[5]$ est introduite :

$$
\begin{aligned}
\varepsilon_{i j} & =\varepsilon_{i j}^{\mathrm{el}}+\varepsilon_{i j}^{\mathrm{r}} \\
D_{i} & =P_{i}^{\mathrm{el}}+P_{i}^{\mathrm{r}}
\end{aligned}
$$

$\varepsilon_{i j}^{\text {el }}$ et $P_{i}^{\mathrm{el}}$ correspondent respectivement à la déformation et la polarisation piézoélectriques qui deviennent nulles lorsqu'il n'y a plus de chargement tandis que $\varepsilon_{i j}^{\mathrm{r}}$ et $P_{i}^{\mathrm{r}}$ désignent la déformation et la polarisation rémanentes. Les parties « élastiques » de la déformation et de la polarisation sont liées par deux relations [6] :

$$
\begin{aligned}
\sigma_{i j} & =C_{i j k l} \varepsilon_{k l}^{\mathrm{el}}-e_{k i j} E_{k} \\
P_{i}^{\mathrm{el}} & =e_{i k l} \varepsilon_{k l}^{\mathrm{el}}+\kappa_{i j} E_{j}
\end{aligned}
$$

$\sigma_{i j}$ et $E_{j}$ désignent les composantes du tenseur des contraintes et du vecteur champ électrique. $C_{i j k l}$ et $\kappa_{i j}$ sont les composantes respectivement des tenseurs d'élasticité et de permittivité diélectrique. Pour la simplification du modèle, ces tenseurs sont supposés isotropes. $e_{i k l}$ est une composante du tenseur d'ordre 3 de 


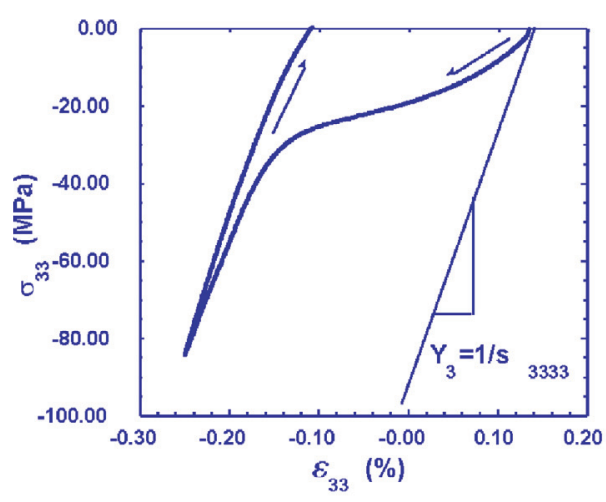

(a)

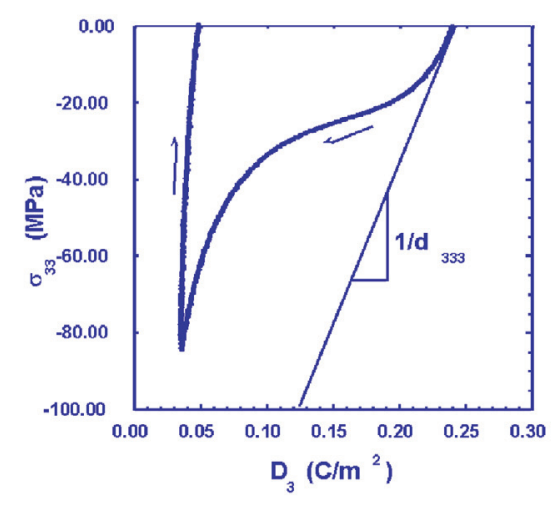

(b)

Fig. 2. Compression d'une céramique de $P Z T$ initialement polarisée : (a) évolution de la déformation en fonction de la contrainte appliquée; (b) évolution de la polarisation totale en fonction de la contrainte [2].

piézoélectricité inverse lié au tenseur de piézoélectricité directe $d_{i m n}$ au travers du tenseur d'élasticité $C_{k l m n}$ tel que

$$
e_{i k l}=C_{k l m n} d_{i m n}
$$

avec

$$
\begin{array}{r}
d_{i m n}=\frac{\sqrt{P_{k}^{r} P_{k}^{r}}}{P_{\mathrm{sat}}}\left\{d_{33} e_{i}^{\mathrm{p}} e_{m}^{\mathrm{p}} e_{n}^{\mathrm{p}}+d_{31}\left(\delta_{m n}-e_{m}^{\mathrm{p}} e_{n}^{\mathrm{p}}\right) e_{i}^{\mathrm{p}}\right. \\
\left.\quad+d_{15}\left[\left(\delta_{i m}-e_{i}^{\mathrm{p}} e_{m}^{\mathrm{p}}\right) e_{n}^{\mathrm{p}}+\left(\delta_{i n}-e_{i}^{\mathrm{p}} e_{n}^{\mathrm{p}}\right) e_{m}^{\mathrm{p}}\right]\right\}
\end{array}
$$

Le tenseur piézoélectrique est isotrope tranverse avec la direction de polarisation de composante $e_{i}^{\mathrm{p}}$ comme direction d'anisotropie. $\varepsilon^{\text {sat }}$ et $P^{\text {sat }}$ sont la déformation et polarisation rémanentes maximales de la céramique tandis que $\delta_{m n}$ désigne le symbole de Kronecker. $d_{33}, d_{31}$ et $d_{15}$ sont les coefficients piézoélectriques classiques pour un matériau pleinement polarisé. En outre, une dépendance linéaire entre les coefficients piézoélectriques et la polarisation rémanente a été mise en évidence par Lynch [2]. Ceci explique que le tenseur dépende de la norme de la polarisation rémanente. À l'échelle macroscopique du polycristal, la déformation rémanente totale n'est pas liée entièrement à la polarisation rémanente macroscopique. C'est la raison pour laquelle nous introduisons une partition de la déformation rémanente [7] :

$$
\varepsilon_{i j}^{\mathrm{r}}=\varepsilon_{i j}^{\mathrm{p}}+\varepsilon_{i j}^{\mathrm{f}}
$$

où $\varepsilon_{i j}^{\mathrm{p}}$ est la partie de la déformation rémanente liée totalement à la polarisation rémanente. Les données expérimentales indiquent que la ferroélasticité et la ferroélectricité sont des comportements n'engendrant pas de variation de volume; par conséquent, le tenseur des déformations est purement déviatorique [8] :

$$
\varepsilon_{i j}^{\mathrm{p}}=\frac{3}{2} \varepsilon^{\mathrm{sat}} \frac{\sqrt{P_{k}^{\mathrm{r}} P_{k}^{\mathrm{r}}}}{P^{\mathrm{sat}}}\left(e_{i}^{\mathrm{p}} e_{j}^{\mathrm{p}}-\frac{1}{3} \delta_{i j}\right)
$$

Nous appelons la déformation rémanente $\varepsilon_{i j}^{\mathrm{f}}$ déformation ferroélastique. Il ne faut pas y voir une décompositon entre l'effet mécanique et l'effet électrique.
En effet, la polarisation et donc la déformation rémanente $\varepsilon_{i j}^{\mathrm{p}}$ sont fonction de l'état mécanique et de l'état électrique. Une contrainte mécanique peut dépolariser le matériau [9]; c'est pourquoi nous introduisons une partition de la polarisation rémanente :

$$
P_{i}^{\mathrm{r}}=P_{i}^{\mathrm{e}}+P_{i}^{\sigma}
$$

où $P_{i}^{\sigma}$ est la composante du vecteur de dépolarisation causée par la contrainte. En tenant compte de ces décompositions, nous choisissons comme variables internes $P_{i}^{\mathrm{e}}$ et $\varepsilon_{i j}^{\mathrm{f}}$. En outre, nous postulons l'existence d'un potentiel thermodynamique duquel dérivent les lois d'état. Les conditions de stabilité thermodynamique sont vérifiées pour un potentiel qui est une fonction concave par rapport à la température, convexe par rapport aux autres variables d'états. Nous pouvons travailler de manière équivalente avec différents potentiels thermodynamiques. Le potentiel énergie libre spécifique de Gibbs $\Psi$ peut être utilisé. Ce potentiel intervient dans l'écriture du second principe de la thermodynamique qui mène à l'inégalité de Clausius-Duhem :

$$
\sigma_{i j} \dot{\varepsilon}_{i j}+E_{i} \dot{D}_{i}-\rho(\dot{\Psi}+s \dot{T})-\frac{q_{i} T_{, i}}{T} \geq 0
$$

où $\rho, s, T$ et $q$ désignent respectivement la masse volumique, l'entropie massique, la température et le flux de chaleur reçu par la céramique. Celui-ci est une fonction des variables

$$
\Psi=\Psi\left(\varepsilon, D, T, \varepsilon^{\mathrm{el}}, \varepsilon^{\mathrm{r}}, P^{\mathrm{el}}, P^{\mathrm{r}}\right)
$$

En fait, les déformations et la polarisation n'interviennent que sous la forme de leurs partitions $\varepsilon-\varepsilon^{\mathrm{r}}=\varepsilon^{\mathrm{el}}$ et $D-P^{\mathrm{r}}=P^{\mathrm{el}}$, soit

$$
\Psi=\Psi\left(\left[\varepsilon-\varepsilon^{\mathrm{r}}\right],\left[D-P^{\mathrm{r}}\right], T\right)=\Psi\left(T, \varepsilon^{\mathrm{el}}, P^{\mathrm{el}}\right)
$$

L'intérêt de cette écriture réside dans les égalités qui en découlent, notamment à partir des partitions de $\varepsilon^{\mathrm{r}}$ et $P^{\mathrm{r}}$, c'est-à-dire

$$
\frac{\partial \Psi}{\partial \varepsilon^{\mathrm{el}}}=\frac{\partial \Psi}{\partial \varepsilon}=-\frac{\partial \Psi}{\partial \varepsilon^{\mathrm{f}}}
$$


et

$$
\frac{\partial \Psi}{\partial P^{\mathrm{el}}}=\frac{\partial \Psi}{\partial D}=-\frac{\partial \Psi}{\partial P^{\mathrm{e}}}
$$

La différentiation du potentiel aboutit à

$$
\dot{\Psi}=\frac{\partial \Psi}{\partial P_{i}^{\mathrm{el}}} \dot{P}_{i}^{\mathrm{el}}+\frac{\partial \Psi}{\partial \varepsilon_{i j}^{\mathrm{el}}} \dot{\varepsilon}_{i j}^{\mathrm{el}}+\frac{\partial \Psi}{\partial T} \dot{T}
$$

L'inégalité de Clausius-Duhem (8) est utilisée avec la relation précédente

$$
\begin{aligned}
\left(\sigma_{i j}-\rho \frac{\partial \Psi}{\partial \varepsilon_{i j}^{\mathrm{el}}}\right) & \dot{\varepsilon}_{i j}^{\mathrm{el}}+\sigma_{i j} \dot{\varepsilon}_{i j}^{\mathrm{r}}+\left(E_{i}-\rho \frac{\partial \Psi}{\partial P_{i}^{\mathrm{el}}}\right) \dot{P}_{i}^{\mathrm{el}} \\
& +E_{i} \dot{P}_{i}^{\mathrm{r}}-\rho\left(s+\frac{\partial \Psi}{\partial T}\right) \dot{T}-\frac{q_{i} T_{, i}}{T} \geq 0
\end{aligned}
$$

Cette écriture permet d'obtenir les lois de la thermopiézoélectricité [3]

$$
\begin{aligned}
\sigma_{i j} & =\rho \frac{\partial \Psi}{\partial \varepsilon_{i j}^{\mathrm{el}}} \\
E_{i} & =\rho \frac{\partial \Psi}{\partial P_{i}^{\mathrm{el}}} \\
s & =-\frac{\partial \Psi}{\partial T}
\end{aligned}
$$

En tenant compte de ces lois, l'inégalité de ClausiusDuhem devient

$$
\Phi=\sigma_{i j} \dot{\varepsilon}_{i j}^{\mathrm{r}}+E_{i} \dot{P}_{i}^{\mathrm{r}}-\frac{q_{i} T_{, i}}{T} \geq 0
$$

Le terme restant dans l'inégalité représente une énergie dissipée. Il correspond à un produit des forces thermodynamiques $\sigma_{i j}, E_{i}$ et $T_{, i}$ avec leurs variables duales qui sont des variables flux : $\dot{\varepsilon}^{\mathrm{r}}$, $\dot{P}^{\mathrm{r}}$ et $\frac{\vec{q}}{T}$. Cette dissipation rend la transformation irréversible : elle crée ainsi de l'entropie et est généralement dissipée sous forme de chaleur. Elle peut être scindée en deux termes. Le premier terme est une dissipation de nature mécanique $\Phi^{\text {mec }}$ avec

$$
\Phi^{\mathrm{mec}}=\sigma_{i j} \dot{\varepsilon}_{i j}^{\mathrm{r}}
$$

La seconde partie est une dissipation de nature électrique $\Phi^{\text {elec }}$ avec

$$
\Phi^{\text {elec }}=E_{i} \dot{P}_{i}^{\mathrm{r}}
$$

Le dernier terme est lié aux pertes dues à la conduction de chaleur

$$
\Phi^{\text {th }}=-\frac{q_{i} T_{, i}}{T}
$$

La partition de la polarisation et la déformation rémanentes permet de réécrire cette dissipation sous une autre forme

$$
\Phi=\sigma_{i j} \dot{\varepsilon}_{i j}^{\mathrm{p}}+E_{i} \dot{P}_{i}^{\mathrm{e}}+\sigma_{i j} \dot{\varepsilon}_{i j}^{\mathrm{f}}+E_{i} \dot{P}_{i}^{\sigma}-\frac{q_{i} T_{, i}}{T} \geq 0
$$

En termes de mécanismes, on peut distinguer plusieurs contributions à la dissipation : celle de la réorientation ferroélectrique au travers des termes

$$
\Phi^{\mathrm{E}}=\sigma_{i j} \dot{\varepsilon}_{i j}^{\mathrm{p}}+E_{i} \dot{P}_{i}^{\mathrm{e}}
$$

La réorientation ferroélastique se retrouve dans les termes suivants

$$
\Phi^{\sigma}=\sigma_{i j} \dot{\varepsilon}_{i j}^{\mathrm{f}}+E_{i} \dot{P}_{i}^{\sigma}
$$

Cette dissipation globale rend compte du caractère hystérétique de ces comportements. Du point de vue macroscopique, il se traduit par les courbes d'évolution du déplacement électrique et de la déformation en fonction du champ électrique. Le comportement ferroélectrique, dont la caractéristique principale est la possibilité d'inverser le sens de la polarisation rémanente, met en jeu plusieurs phénomènes physiques : la croissance de domaines antiparallèles, le mouvement de parois de domaines et la croissance de nouveaux domaines antiparallèles. Tous ces mécanismes sont entravés par la présence de défauts et d'imperfections structurales. Les défauts ont un effet inhibateur sur le mouvement de parois de domaines et contribuent à la dissipation [10]. $\Phi$ peut être décomposé en deux termes de dissipation

$$
\Phi=\Phi^{1}+\Phi^{2}
$$

avec

$$
\Phi^{1}=\sigma_{i j} \dot{\varepsilon}_{i j}^{\mathrm{f}}+E_{i} \dot{P}_{i}^{\mathrm{e}}-\frac{q_{i} T_{, i}}{T} \quad \Phi^{2}=\sigma_{i j} \dot{\varepsilon}_{i j}^{\mathrm{p}}+E_{i} \dot{P}_{i}^{\sigma}
$$

L'inégalité à vérifier est celle de Clausius-Duhem $\Phi \geq$ 0 . Une condition forte et suffisante est $\Phi^{1} \geq 0$ et $\Phi^{2} \geq 0$. Compte tenu du caractère dissipatif de la transformation, nous postulons l'existence d'un potentiel de dissipation $\varphi$ fonction des variables flux $\dot{\varepsilon}^{\mathrm{f}}$, $\dot{P}^{\mathrm{e}}$ et $\frac{\vec{q}}{T}$. Ce potentiel est une fonction convexe, quasi-homogène, positive et nulle à l'origine dans l'espace des variables flux. Les lois complémentaires s'expriment alors par la propriété de normalité

$$
\sigma_{i j}=\frac{\partial \varphi}{\partial \dot{\varepsilon}_{i j}^{\mathrm{f}}} \quad E_{i}=\frac{\partial \varphi}{\partial \dot{P}_{i}^{\mathrm{e}}} \quad T_{, i}=-\frac{\partial \varphi}{\partial\left(q_{i} / T\right)}
$$

Les forces thermodynamiques sont les composantes du vecteur $\operatorname{grad} \varphi$. L'objectif est de fournir des lois d'évolution pour les variables internes. Il est donc plus commode d'exprimer ces lois complémentaires sous la forme de lois d'évolution des variables flux $\dot{\varepsilon}^{\mathrm{f}}$, $\dot{P}^{\mathrm{e}}$ et $\frac{\vec{q}}{T}$ en fonction des variables duales. La transformée de Legendre-Fenchel permet de définir le potentiel correspondant $\varphi^{*}\left(\sigma_{i j}, E_{i}, T_{, i}\right)$ dual de $\varphi$ par rapport aux variables. Cette transformation s'écrit

$$
\begin{aligned}
\varphi^{*}\left(\sigma_{i j}, E_{i}, T_{, i}\right)=\operatorname{Sup}_{\left(\dot{\varepsilon}^{\mathrm{f}}, \dot{P}^{E}, \frac{q_{i}}{T}\right)} & \left(\left[\sigma_{i j} \dot{\varepsilon}_{i j}^{\mathrm{f}}+E_{i} \dot{P}_{i}^{\mathrm{e}}-\frac{q_{i} T_{, i}}{T}\right]\right. \\
\left.-\varphi\left(\dot{\varepsilon}_{i j}^{\mathrm{f}}, \dot{P}_{i}^{\mathrm{e}}, \frac{q_{i} T_{, i}}{T}\right)\right) & (28)
\end{aligned}
$$


Les propriétés de normalité sont conservées pour $\dot{\varepsilon}^{\mathrm{f}}, \quad \dot{P}^{\mathrm{e}}$ et $\frac{\vec{q}}{T}$ si $\varphi^{*}$ est dérivable. $\varphi^{*}$ possède les mêmes propriétés que $\varphi$, c'est-à-dire d'être convexe, quasi-homogène, positive et nulle à l'origine. Les lois complémentaires s'écrivent alors

$$
\dot{\varepsilon}_{i j}^{\mathrm{f}}=\frac{\partial \varphi^{*}}{\partial \sigma_{i j}} \quad \dot{P}_{i}^{\mathrm{e}}=\frac{\partial \varphi^{*}}{\partial E_{i}} \quad-\frac{q_{i}}{T}=\frac{\partial \varphi^{*}}{\partial\left(T_{, i}\right)}
$$

Elles peuvent se réécrire [11] :

$$
\dot{\varepsilon}_{i j}^{\mathrm{f}}=\dot{\lambda}^{\sigma} \frac{\partial f^{\sigma}}{\partial \sigma_{i j}} \quad \text { si } \quad\left\{\begin{array}{l}
f^{\sigma}=0 \\
\dot{f}^{\sigma}=0
\end{array}\right.
$$

et

$$
\dot{P}_{i}^{\mathrm{e}}=\dot{\lambda}^{\mathrm{e}} \frac{\partial f^{\mathrm{e}}}{\partial E_{i}} \quad \text { si } \quad\left\{\begin{array}{l}
f^{\mathrm{e}}=0 \\
\dot{f}^{\mathrm{e}}=0
\end{array}\right.
$$

où $f^{\sigma}$ et $f^{\mathrm{e}}$ sont des fonctions critères convexes respectivement en $\sigma$ et en $E$. $\dot{\lambda}^{\sigma}$ et $\dot{\lambda}^{\mathrm{e}}$ sont des multiplicateurs que nous qualifierons de ferroélastique et ferroélectrique. Les conditions de consistances $\dot{f}^{\sigma}=0$ et $\dot{f}^{\mathrm{e}}=0$ permettent de déterminer leurs expressions. Dans la suite, nous allons exprimer explicitement ces fonctions. Elles fourniront les lois d'évolution des deux variables internes $\varepsilon^{\mathrm{f}}$ et $P^{\mathrm{e}}$.

\subsubsection{Surfaces de charge ferroélectrique et ferroélastique}

Comme il a été mentionné précédemment, les comportements ferroélectrique et ferroélastique proviennent de la rotation de domaines uniformément polarisés lorsque la contrainte mécanique ou le champ électrique atteignent et dépassent les valeurs coercitives. En dessous de ces valeurs, le comportement est piézoélectrique. Les courbes expérimentales peuvent être décrites par un écrouissage cinématique linéaire. Par conséquent, nous introduisons une fonction de charge ferroélastique :

$$
f^{\sigma}=\sqrt{\frac{3}{2}\left(s_{i j}-\alpha_{i j}\right)\left(s_{i j}-\alpha_{i j}\right)}-\widehat{\sigma}_{\mathrm{c}}
$$

où $s_{i j}$ et $\alpha_{i j}$ sont respectivement les composantes du déviateur des contraintes et d'un tenseur d'écrouissage. $\widehat{\sigma_{c}}$ est une valeur critique dépendant du champ électrique. Nous supposons qu'elle est proportionnelle à la projection du vecteur champ électrique suivant la direction de polarisation $\overrightarrow{e^{\mathrm{p}}}$ :

$$
\widehat{\sigma_{\mathrm{c}}}=\sigma_{\mathrm{c}}+n E_{i} e_{i}^{\mathrm{p}}
$$

où $\sigma_{\mathrm{c}}$ et $n$ sont la contrainte coercitive et un paramètre du matériau. Il est possible de choisir la fonction $f^{\sigma}$ comme un potentiel duquel dérivent les lois d'évolution de la variable interne $\varepsilon_{i j}^{\mathrm{f}}$ et donc celle de la variable d'écrouissage $\alpha_{i j}$ dans le cas d'un écrouissage cinématique linéaire [12]. La loi de normalité s'écrit alors :

$$
\dot{\varepsilon}_{i j}^{\mathrm{f}}=\dot{\lambda}^{\sigma} \frac{\partial f^{\sigma}}{\partial \sigma_{i j}}
$$

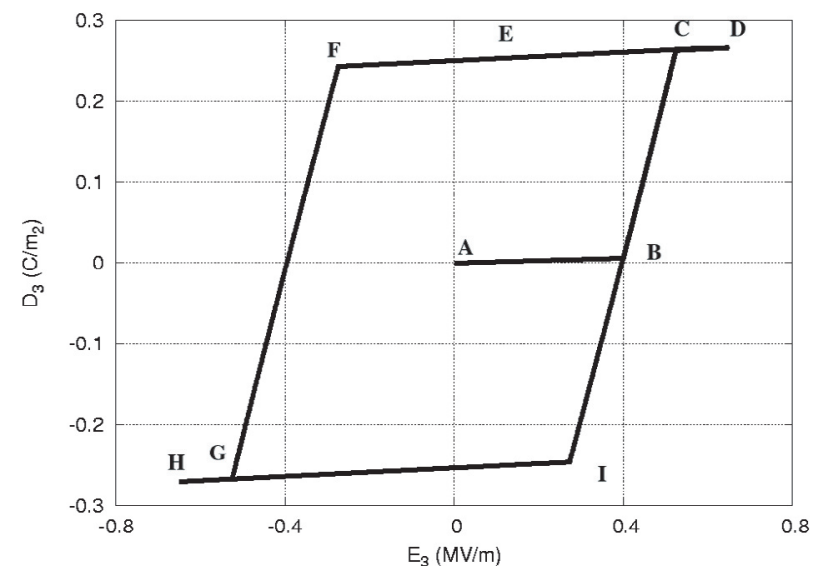

Fig. 3. Évolution du déplacement électrique en fonction du champ électrique. Initialement, le matériau est non polarisé. À partir du champ coercitif, le matériau se polarise jusqu'à saturation.

où $\dot{\lambda}^{\sigma}$ est un multiplicateur ferroélastique déterminé par la relation de consistance $\left(\dot{f}^{\sigma}=0\right)$. L'écrouissage cinématique linéaire s'exprime ainsi :

$$
\dot{\alpha}_{i j}=\frac{2}{3} h \dot{\varepsilon}_{i j}^{\mathrm{f}}
$$

où $h$ est un paramètre du matériau. De même, nous introduisons une fonction de charge ferroélectrique :

$$
f^{\mathrm{e}}=\sqrt{\left(E_{i}-X_{i}\right)\left(E_{i}-X_{i}\right)}-E_{\mathrm{c}}
$$

$X_{i}$ est une composante d'un vecteur d'écrouissage et $E_{\mathrm{c}}$ est le champ électrique coercitif. La loi de normalité définit la loi d'évolution de la variable interne $P_{i}^{\mathrm{e}}$ :

$$
\dot{P}_{i}^{\mathrm{e}}=\dot{\lambda}^{\mathrm{e}} \frac{\partial f^{\mathrm{e}}}{\partial E_{i}}
$$

où $\dot{\lambda}^{\text {e }}$ est un multiplicateur ferroélectrique déterminé par la relation de consistance $\left(\dot{f}^{\mathrm{e}}=0\right)$. L'écrouissage cinématique linéaire permet d'écrire :

$$
\dot{X}_{i}=\beta \dot{P}_{i}^{\mathrm{e}}
$$

avec $\beta$ un paramètre du matériau. Dans le cas de la dépolarisation mécanique, l'évolution de $P_{i}^{\sigma}$ est donnée par la loi d'évolution de $\varepsilon_{i j}^{\mathrm{f}}$ telle que :

$$
\dot{P}_{i}^{\sigma}=\frac{P^{\mathrm{sat}}}{\varepsilon^{\mathrm{sat}}} \dot{\varepsilon}_{i j}^{\mathrm{f}} e_{i}^{\mathrm{p}}
$$

\section{Résultats}

Plusieurs cas de charges tels que des cyclages mécaniques de traction et compression ainsi que des cyclages électriques de polarisation et dépolarisation uniaxiales ont été simulés. À titre d'exemple, les figures 3 et 4 montrent les différents hystérésis après un cyclage électrique. En outre, après avoir polarisé électriquement 


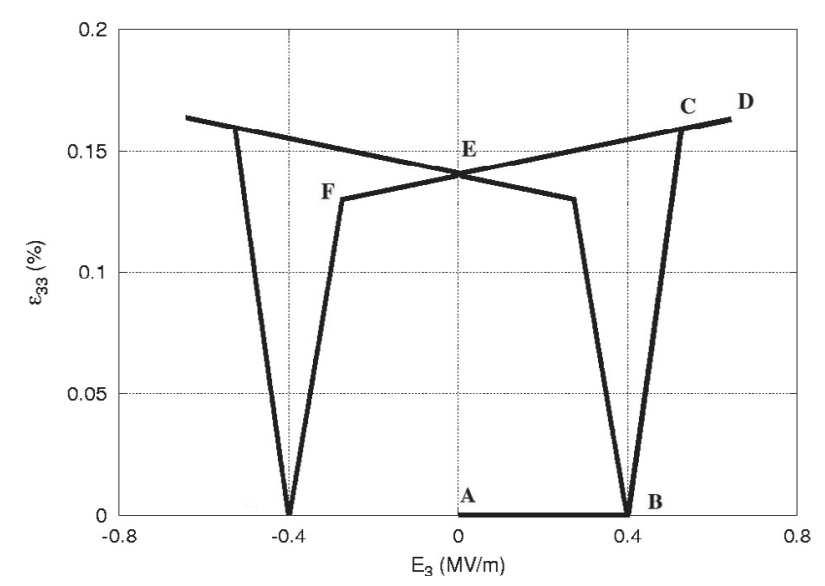

Fig. 4. Butterfly électrique : évolution de la déformation en fonction du champ électrique.

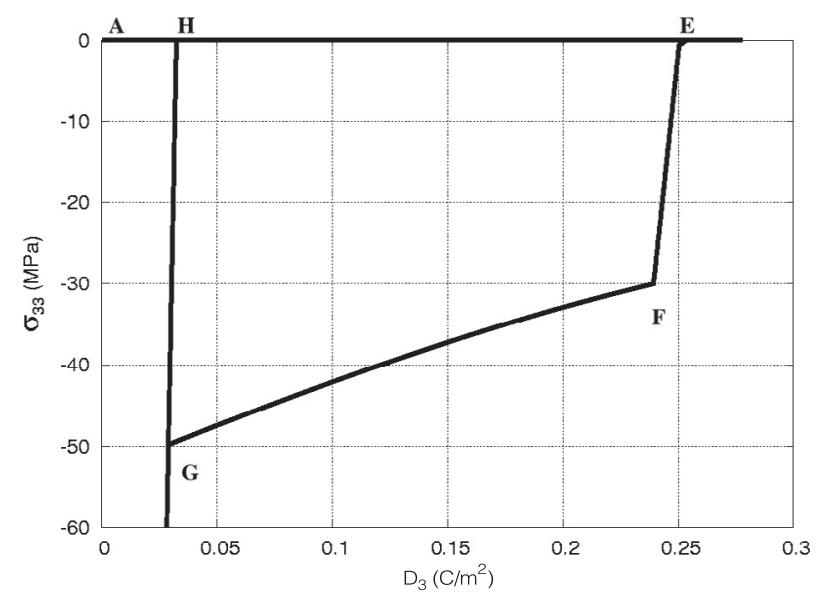

Fig. 5. Dépolarisation mécanique : le comportement piézoélectrique est suivi de la ferroélasticité puis redevient à nouveau piézoélectrique.

le matériau, il est possible de le dépolariser en le soumettant à une contrainte de compression (Fig. 5).

Les courbes expérimentales montrent que la dépolarisation mécanique, qui est due à la réorientation des domaines dans des directions aléatoires, n'aboutit pas à une dépolarisation totale du matériau. Il subsiste toujours une polarisation rémanente non nulle. C'est la raison pour laquelle, lors d'un essai de compression sur un matériau polarisé, le comportement est initiallement piézoélectrique, puis ferroélectrique et redevient piézoélectrique.

\section{Conclusions}

Ce modèle basé sur l'utilisation de variables internes et de fonctions de charge similaires aux fonctions de charge élasto-plastique va être implémenté dans un code élément fini. Cette étape nécessite la définition d'un élément fini ayant les degrés de liberté adéquats afin de prendre en compte ces comportements spécifiques.

\section{Références}

[1] M.E. Lines, A.M. Glass, Principles and applications of ferroelectrics and related materials, Oxford University Press, Oxford, 1997

[2] C.S. Lynch, The effect of uniaxial stress on the electromechanical response of $8 / 65 / 35$ plzt, Acta Mater. 46 (1996) 5085-5094

[3] M. Elhadrouz, T. Ben Zineb, É. Patoor, Constitutive law for ferroelastic and ferroelectric piezoceramics, J. Int. Mat. Sys. Struc. (2004), in press

[4] J. Lemaitre, J.-L. Chaboche, Mechanics of solid materials, Cambridge University Press, Cambridge, 1990

[5] E. Bassiouny, A.F. Ghaleb, G.A. Maugin, Thermodynamical formulation for coupled electromechanical hysteresis effects-i. basic equations, Int. J. Eng. Sci. 26 (1988) 1279-1295

[6] J. Grindlay, An introduction to the phenomenological theory of ferroelectricity, Pergamon Press, Oxford, 1970

[7] M. Kamlah, C. Tsakmakis, Phenomenological modeling of the non-linear electromechanical coupling in ferroelectrics, Int. J. Solids Struct. 36 (1999) 669-695

[8] H. Cao, A.G. Evans, Nonlinear deformation of ferroelectric ceramics, J. Am. Ceram. Soc. 76 (1993) 890-896

[9] A. Schaufele, K.H. Hardtl, Ferroelastic properties of lead zirconate titanate ceramics, J. Am. Ceram. Soc. 79 (1996) $2637-2640$

[10] C.S. Damjanovic, Piezoelectric properties of perovskite ferroelectrics: unsolved problems and future research, An. Chi. Sci. Mater. 26 (1998) 99-106

[11] M. Elhadrouz, Modélisation du comportement des céramiques ferroélectriques et ferroélastiques, Ph.D. Thèse, École nationale supérieure d'arts et métiers, France, 2004

[12] M.E. Lines, A.M. Glass, Mechanics of deformable solids, Springer, Berlin, 2000 